Research Article

\title{
Plane Waves Scattered by a Spherical Inclusion in a Half Space of Poroelastic Material
}

\author{
Doo-Sung Lee * \\ Department of Mathematics, College of Education, Konkuk University, Seoul, Korea; E-Mail: \\ dslee@konkuk.ac.kr
}

* Correspondence: Doo-Sung Lee; E-Mail: dslee@konkuk.ac.kr

Academic Editor: Vardan Apinyan

Special Issue: Quantum Mechanics in Solid State Systems

Recent Progress in Materials

2021, volume 3 , issue 2

doi:10.21926/rpm.2102021
Received: December 09, 2020

Accepted: May 05, 2021

Published: June 02, 2021

\begin{abstract}
This paper concerns a poroelastic half-space in which plane compressional waves are scattered by a spherical inclusion. Addition theorems for the spherical wave functions are utilized to meet the boundary conditions on the plane, and the satisfaction of the given conditions on the boundary of the sphere leads to three infinite series equations, whose solution can be acquired by successive approximations. Further, its existence and uniqueness are discussed.
\end{abstract}

\section{Keywords}

Half space; poroelastic material; spherical cavity; scattering; elliptic equation

\section{Introduction}

The use of the Biot formulation of a porous medium is well cited in the literature; see examples [1-5]. Some of the works in this area have also sought to use the Biot formulation of a poroelastic

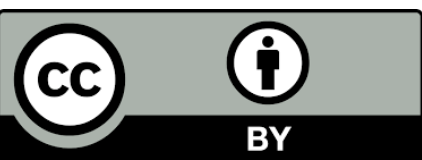

(C) 2021 by the author. This is an open access article distributed under the conditions of the Creative Commons by Attribution License, which permits unrestricted use, distribution, and reproduction in any medium or format, provided the original work is correctly cited. 
material to determine the bone rigidity coefficients. Buchnan and Gilbert $[6,7]$ used the Biot formulation of poroelastic material to determine bone rigidity coefficients by Gilbert et al. [8, 9]. In a recent work, Lee [10] considered the scattering of the plane compressional waves by two rigid inclusions in an infinite poroelastic medium.

It is well known that Biot's theory predicts a fast and slow compressional wave. Since the second compressional wave does not occur for a purely elastic material, the detection of the two different compressional waves indicates the poroelastic nature of the specimen. Hosokawa and Otani [11] studied the use of the Biot formulation of bone. Additionally, they also identified both fast and slow waves in the cancellous bone.

In this paper, Biot's equation of motion is solved analytically when a plane, compressional wave impinges on a rigid spherical inclusions in a poroelastic half space. The incident wave is composed of a linear combination of the Biot "fast" and "slow" waves. An image method is applied to Biot's equation of motion to obtain three infinite series equations, using an equation [12], and subsequently, a successive approximation technique is employed to obtain the solution. The existence and uniqueness of this solution are discussed below.

\section{Biot Equations}

The homogeneous media Biot equations for the displacements have been given earlier $[13,14]$ as follows:

$$
\begin{aligned}
& \nabla \cdot \vec{\sigma}_{s}-b \frac{\partial}{\partial t}(\vec{U}-\vec{V})=\rho_{11}^{\prime} \frac{\partial^{2} \vec{U}}{\partial t^{2}}+\rho_{12}^{\prime} \frac{\partial^{2} \vec{V}}{\partial t^{2}} \\
& \nabla s+b \frac{\partial}{\partial t}(\vec{U}-\vec{V})=\rho_{12}^{\prime} \frac{\partial^{2} \vec{U}}{\partial t^{2}}+\rho_{22}^{\prime} \frac{\partial^{2} \vec{V}}{\partial t^{2}}
\end{aligned}
$$

where position $(\vec{x})$ and time $(\mathrm{t})$ are two independent parameters, $\vec{U}(\vec{x}, t)$ represents the solid displacement, $\vec{V}(\vec{x}, t)$ represents the fluid displacement, and $\vec{\sigma}_{s}$ is the defined solid stress tensor so that $\vec{\sigma}_{s} \vec{n}$ is the force, per unit area of the bulk material, applied to the solid part of the material. Additionally, $s$ is the negative of the pore pressure $\left(p_{p}\right)$ times void volume fraction $f$ of the material. The total stress is given by $\vec{\sigma}=\vec{\sigma}_{s}+s \vec{I}$, where $\vec{I}$ is the identity tensor.

For the rest of the analysis, we will be considering the case of harmonic motion. Hence, the displacements can be expressed as:

$$
\begin{aligned}
& \vec{U}(\vec{x}, t)=\vec{u}(\vec{x}) \exp (-i \omega t) \\
& \vec{V}(\vec{x}, t)=\vec{v}(\vec{x}) \exp (-i \omega t)
\end{aligned}
$$

where $\omega$ is the circular frequency. The Biot equations have too many unknown functions when both the fluid and the solid displacements are used. Therefore, it is better just to use the solid displacement and the fluid pressure [15]. To see how this is done, we recall the constitutive equations for a Biot-type material, which are given by:

$$
\vec{\sigma}:=\left(\lambda+\frac{Q^{2}}{R}\right) \nabla \cdot \vec{u} I+\mu\left(\nabla \vec{u}+(\nabla \vec{u})^{T}\right)+Q \nabla \cdot \vec{v} I+s I,
$$


where,

$$
s:=Q \nabla \cdot \vec{u}+R \nabla \cdot \vec{v} .
$$

The second of the above equations can be used to eliminate the fluid displacement from the equations of motion. To this end, we derive an equation that is satisfied by $s$ by first obtaining from equation (2):

$$
\nabla s+\omega^{2}\left(\rho_{12} \vec{u}+\rho_{22} \vec{v}\right)=0,
$$

where $\rho_{12}=\rho_{12}^{\prime}-\frac{i b}{\omega}, \rho_{22}=\rho_{22}{ }^{\prime}+\frac{i b}{\omega}$ and then

$$
\triangle s+\frac{\omega^{2} \rho_{22}}{R} s+\omega^{2} \rho_{22}\left(\frac{\rho_{12}}{\rho_{22}}-\frac{Q}{R}\right) \nabla \cdot \vec{u}=0 .
$$

Following an earlier study [15], we identify a pressure $p$ given by:

$$
p:=-\frac{s}{\omega \sqrt{\rho_{22}}} .
$$

Now from equation (4), this pressure can be seen to satisfy the equation:

$$
\triangle p+\beta \omega^{2} p-\alpha \nabla \cdot \vec{u}=0,
$$

where,

$$
\alpha:=\sqrt{\rho_{22}} \omega\left(\frac{\rho_{12}}{\rho_{22}}-\frac{Q}{R}\right), \text { and } \beta:=\frac{\rho_{22}}{R} .
$$

From equations ( 3 ) and (5), the fluid displacement $\vec{v}$ can be given in terms of the solid displacement $\vec{u}$ and the scaled pore pressure $p$ as:

$$
\vec{v}=\frac{\nabla p}{\omega \sqrt{\rho_{22}}}-\frac{\rho_{12} \vec{u}}{\rho_{22}} .
$$

If $\sigma_{s}:=\sigma-s I$, then the other equation of motion (1) may be written as:

$$
\nabla \cdot \vec{\sigma}_{s}+\omega^{2}\left(\rho_{11} \vec{u}+\rho_{12} \vec{v}\right)=0
$$

where, $\rho_{11}=\rho_{11}{ }^{\prime}+\frac{i b}{\omega}$. Further, by eliminating the water displacement vector $\vec{v}$, we obtain the form:

$$
\nabla \cdot \vec{\sigma}_{s}+\omega^{2}\left(\rho_{11}-\frac{\rho_{12}^{2}}{\rho_{22}}\right) \vec{u}-\frac{\rho_{12}}{\rho_{22}} \nabla s=0
$$

which simplifies to the form:

$$
(\lambda+\mu) \nabla(\nabla \cdot \vec{u})+\mu \nabla \cdot \nabla \vec{u}+\alpha \nabla p+\omega^{2} \rho \vec{u}=0 .
$$


Following Zimmerman and Stern, we use the representation of solid displacement and pressure as given by:

$$
\begin{array}{cc}
\vec{u}= & \nabla \phi_{f}+\nabla \phi_{s}+\nabla \wedge \nabla \wedge \vec{\psi} \\
p= & A_{f} \Delta \phi_{f}+A_{s} \Delta \phi_{s},
\end{array}
$$

Substituting these into equations (6) and (8), we obtain the equations for the scalar and vector potentials. These are [15].

$$
\begin{gathered}
a_{f} \Delta \phi_{f}+\omega^{2} \rho \phi_{f}+a_{s} \Delta \phi_{s}+\omega^{2} \rho \phi_{s}=0, \\
A_{f} \Delta \phi_{f}-d_{f} \phi_{f}+A_{s} \Delta \phi_{s}-d_{s} \phi_{s}=0, \\
\left\{\triangle+k_{t}^{2}\right\} \nabla \wedge \vec{\psi}=0 .
\end{gathered}
$$

Here the following notation is used:

$$
\begin{gathered}
a_{f, s}:=\lambda+2 \mu+\alpha A_{f, s}, \quad \rho:=\frac{\rho_{11} \rho_{22}-\rho_{12}^{2}}{\rho_{22}}, \\
d_{f, s}:=A_{f, s} \beta \omega^{2}-\alpha, \quad k_{t}^{2}:=\frac{\rho \omega^{2}}{\mu} .
\end{gathered}
$$

In order to decouple these waves ${ }^{1}$ we require the following conditions to be met:

$$
\frac{d_{f}}{A_{f}}=\frac{\rho \omega^{2}}{a_{f}}, \text { and } \frac{d_{s}}{A_{s}}=\frac{\rho \omega^{2}}{a_{s}} .
$$

This leads to

$$
A_{f, s}:=\frac{-D \pm \sqrt{D^{2}+4(\lambda+2 \mu) \alpha^{2} \beta \omega^{2}}}{2 \alpha \beta \omega^{2}}
$$

where $D:=(\lambda+2 \mu) \beta \omega^{2}-\alpha^{2}-\rho \omega^{2}$. The fast and slow compression waves then satisfy

$$
\triangle \phi_{f, s}+\kappa_{f, s}^{2} \phi_{f, s}=0
$$

where

$$
\kappa_{f, s}^{2}:=\frac{d_{f, s}}{A_{f, s}}=\frac{\rho \omega^{2}}{a_{f, s}} .
$$

Let us suppose that $\psi$ satisfies $\Delta \psi+k_{t}^{2} \psi=0$. If we take $\vec{\psi}=\mathrm{r} \psi e_{r}$ where $\mathrm{e}_{r}=$ unit vector in $r$-direction, then we see that $\vec{\psi}$ satisfies equation (10).

\footnotetext{
${ }^{1}$ Zimmerman and Stern say for reasons of "compatibility".
} 


\section{Boundary Conditions on the Plane}

In this paper, we consider the problem of half space. Hence, we employ both spherical $(r, \theta)$ and cylindrical $(\rho, z)$ coordinates. The center of the spherical inclusion is at $(\rho, z)=(0,0)$, and the plane face of the half space is located at $z=-d$. On the planar surface, the boundary conditions are $u_{z}^{(r)}=\sigma_{\rho z}^{(r)}=0$. Here $(r)$ refers to reflected waves. The incident compressional wave is made up of both fast and slow compression components but no shear component; therefore, the incident wave scalar potential is composed of a fast and a slow contribution. We write:

$$
\phi^{(i)}=\phi_{f}^{(i)}+\phi_{s}^{(i)}
$$

and

$$
\phi_{f}^{(i)}=\phi_{0 f}^{(i)}\left\{e^{i \kappa_{f} z}+e^{-i \kappa_{f}(z+2 d)}\right\}, \phi_{s}^{(i)}=\phi_{0 s}^{(i)}\left\{e^{i \kappa_{s} z}+e^{-i \kappa_{s}(z+2 d)}\right\}
$$

Since the displacements in cylindrical coordinates $[13,14]$ are

$$
\vec{u}=\nabla \phi_{f}+\nabla \phi_{s}+\nabla \wedge(\nabla \wedge \vec{\psi}) \text {. }
$$

If $\psi=0$, then displacements are

$$
\begin{aligned}
& u_{\rho}=\frac{\partial \phi_{f}}{\partial \rho}+\frac{\partial \phi_{s}}{\partial \rho} \\
& u_{z}=\frac{\partial \phi_{f}}{\partial z}+\frac{\partial \phi_{s}}{\partial z} .
\end{aligned}
$$

Therefore, the tangential stress is:

$$
\sigma_{\rho z}=\mu\left(\frac{\partial u_{z}}{\partial \rho}+\frac{\partial u_{\rho}}{\partial z}\right)=2 \mu\left\{\frac{\partial^{2} \phi_{f}}{\partial \rho \partial z}+\frac{\partial^{2} \phi_{s}}{\partial \rho \partial z}\right\}
$$

We see that $u_{z}^{(i)}=\sigma_{\rho z}^{(i)}=0$ at $z=-d$. Thus the boundary conditions on $z=-d$ are

$$
u_{z}^{(i)}+u_{z}^{(r)}=v_{z}^{(i)}+v_{z}^{(r)}=\sigma_{\rho z}^{(i)}+\sigma_{\rho z}^{(r)}=0 \text { at } z=-d
$$

Thus, we use the image method, i.e., we symmetrically extend the status to the region $z<0$, and as a result, the present problem gets converted into a problem of two spheres in a full space. The second sphere is located at $(\rho, z)=(0,-2 d)$ whose coordinate systems are $\left(r^{\prime}, \theta^{\prime}\right)$. 


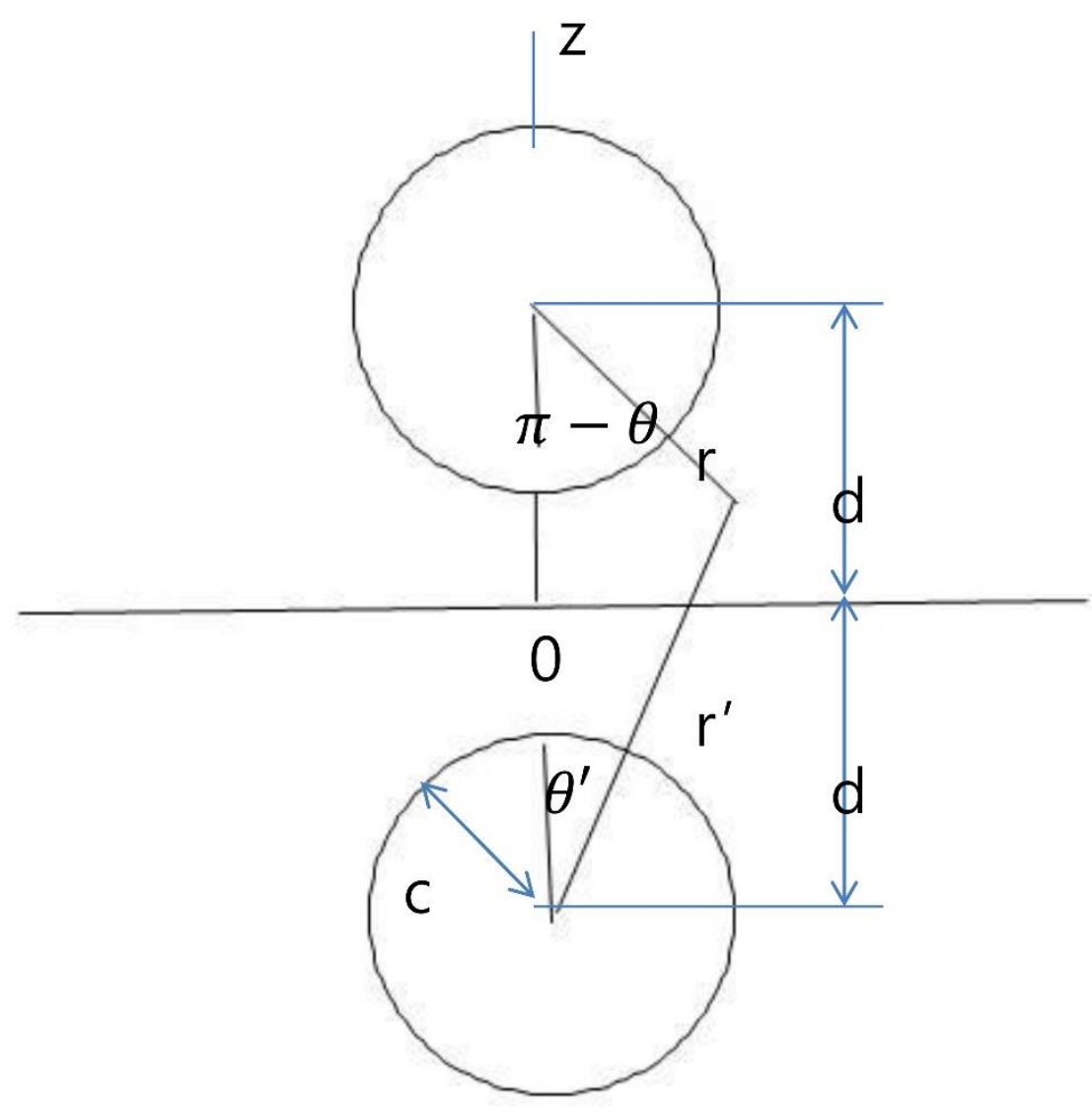

Figure $1 \mathrm{C}$ Configuration of the sphere.

\section{Boundary Conditions on the Sphere}

The appropriate functions for $\phi_{f}, \phi_{s}$, and $\psi$ are:

$$
\phi_{f}=\sum_{n=0}^{\infty} c_{n f} h_{n}^{(1)}\left(\kappa_{f} r\right) P_{n}(\cos \theta)+\sum_{q=0}^{\infty} c^{\prime}{ }_{q f} h_{q}^{(1)}\left(\kappa_{f} r^{\prime}\right) P_{q}\left(\cos \theta^{\prime}\right)
$$

where $h_{n}^{(1)}(r)=\sqrt{\frac{\pi}{2 r}} H_{n+1 / 2}^{(1)}(r)$. Here $\sqrt{\frac{\pi}{2 r}} H_{n+1 / 2}^{(1)}(r)$ is the spherical Bessel function of the third kind. Similarly, we can write:

$$
\phi_{s}==\sum_{n=0}^{\infty} c_{n s} h_{n}^{(1)}\left(\kappa_{s} r\right) P_{n}(\cos \theta)+\sum_{q=0}^{\infty} c^{\prime}{ }_{q s} h_{q}^{(1)}\left(\kappa_{s} r^{\prime}\right) P_{q}\left(\cos \theta^{\prime}\right),
$$

We can further express functions in $\left(r^{\prime}, \theta^{\prime}\right)$ by $(r, \theta)$ using the addition theorems for spherical wave functions. Using the relations:

$$
h_{q}^{(1)}\left(k r^{\prime}\right) P_{q}\left(\cos \theta^{\prime}\right)=\sum_{n=0}^{\infty} Q_{0 q 0 n}^{(2)}(2 d) j_{n}(k r) P_{n}(\cos \theta),
$$


in [12], and the expression $Q_{0 q 0 n}^{(2)}(2 d)$ is given in an earlier study [12]. Thus, equation (17) can be written as:

$$
\phi_{f}=\sum_{n=0}^{\infty}\left[c_{n f} h_{n}^{(1)}\left(\kappa_{f} r\right)+b_{n f} j_{n}\left(\kappa_{f} r\right)\right] P_{n}(\cos \theta)
$$

where

$$
b_{n f}=\sum_{q=0}^{\infty} c^{\prime}{ }_{q f} Q_{q n}
$$

Here, we have used the short-hand notation $Q_{q n}:=Q_{0 q 0 n}^{(2)}(2 d)$.

Likewise, equation (18) can be written as:

$$
\phi_{s}=\sum_{n=0}^{\infty}\left[c_{n s} h_{n}^{(1)}\left(\kappa_{s} r\right)+b_{n s} j_{n}\left(\kappa_{s} r\right)\right] P_{n}(\cos \theta),
$$

where

$$
b_{n s}=\sum_{q=0}^{\infty} c^{\prime}{ }_{q s} Q_{q n}
$$

Also,

$$
\begin{gathered}
\psi=\sum_{n=0}^{\infty} c_{n t} h_{n}^{(1)}\left(k_{t} r\right) P_{n}(\cos \theta)+\sum_{q=0}^{\infty} c^{\prime}{ }_{q t} h_{q}^{(1)}\left(k_{t} r^{\prime}\right) P_{q}\left(\cos \theta^{\prime}\right) \\
=\sum_{n=0}^{\infty}\left[c_{n t} h_{n}^{(1)}\left(k_{t} r\right)+b_{n t} j_{n}\left(k_{t} r\right)\right] P_{n}(\cos \theta)
\end{gathered}
$$

where

$$
b_{n t}=\sum_{q=0}^{\infty} c^{\prime}{ }_{q t} Q_{q n}
$$

The displacements in spherical coordinates are:

$$
\begin{gathered}
u_{r}=\frac{\partial \phi_{f}}{\partial r}+\frac{\partial \phi_{s}}{\partial r}-\frac{1}{r \sin \theta} \frac{\partial}{\partial \theta}\left(\sin \theta \frac{\partial \psi}{\partial \theta}\right), \\
u_{\theta}=\frac{1}{r} \frac{\partial \phi_{f}}{\partial \theta}+\frac{1}{r} \frac{\partial \phi_{s}}{\partial \theta}+\frac{1}{r} \frac{\partial^{2} r \psi}{\partial r \partial \theta}
\end{gathered}
$$


Recent Progress in Materials 2021; 3(2), doi:10.21926/rpm.2102020

$$
v_{r}=\frac{1}{\omega \sqrt{\rho_{22}}}\left(-A_{f} \kappa_{f}^{2} \frac{\partial \phi_{f}}{\partial r}-A_{s} \kappa_{s}^{2} \frac{\partial \phi_{s}}{\partial r}\right)-\frac{\rho_{12}}{\rho_{22}} u_{r}
$$

The last expression is from equation (7) and thus can be written as:

$$
u_{r}^{(r)}=\frac{1}{r} \sum_{n=0}^{\infty}\left[c_{n f} \mathcal{E}_{11}+c_{n s} \mathcal{E}_{12}+c_{n t} \mathcal{E}_{13}+b_{n f} \mathcal{F}_{11}+b_{n s} \mathcal{F}_{12}+b_{n t} \mathcal{F}_{13}\right] P_{n}(\cos \theta)
$$

where

$$
\begin{gathered}
\mathcal{E}_{11}=-\kappa_{f} r h_{n+1}^{(1)}\left(\kappa_{f} r\right)+n h_{n}^{(1)}\left(\kappa_{f} r\right), \\
\mathcal{E}_{12}=-\kappa_{s} r h_{n+1}^{(1)}\left(\kappa_{s} r\right)+n h_{n}^{(1)}\left(\kappa_{s} r\right), \\
\mathcal{E}_{13}=n(n+1) h_{n}^{(1)}\left(k_{t} r\right), \\
\mathcal{F}_{11}=-\kappa_{f} r j_{n+1}\left(\kappa_{f} r\right)+n j_{n}\left(\kappa_{f} r\right), \\
\mathcal{F}_{12}=-\kappa_{s} r j_{n+1}\left(\kappa_{s} r\right)+n j_{n}\left(\kappa_{s} r\right), \\
\mathcal{F}_{13}=n(n+1) j_{n}\left(k_{t} r\right), \\
u_{\theta}^{(r)}=\frac{1}{r} \sum_{n=0}^{\infty}\left[c_{n f} \mathcal{E}_{21}+c_{n s} \mathcal{E}_{22}+c_{n t} \varepsilon_{23}+b_{n f} \mathcal{F}_{21}+b_{n s} \mathcal{F}_{22}+b_{n t} \mathcal{F}_{23}\right] \frac{\partial P_{n}(\cos \theta)}{\partial \theta},
\end{gathered}
$$

where

$$
\begin{gathered}
\mathcal{E}_{21}=h_{n}^{(1)}\left(\kappa_{f} r\right), \mathcal{E}_{22}=h_{n}^{(1)}\left(\kappa_{s} r\right), \mathcal{E}_{23}=-k_{t} r h_{n+1}^{(1)}\left(k_{t} r\right)+(n+1) h_{n}^{(1)}\left(k_{t} r\right), \\
\mathcal{F}_{21}=j_{n}\left(\kappa_{f} r\right), \mathcal{F}_{22}=j_{n}\left(\kappa_{s} r\right), \mathcal{F}_{23}=-k_{t} r j_{n+1}\left(k_{t} r\right)+(n+1) j_{n}\left(k_{t} r\right), \\
v_{r}^{(r)}=\frac{1}{r} \sum_{n=0}^{\infty}\left[c_{n f} \mathcal{E}_{31}+c_{n s} \mathcal{E}_{32}+c_{n t} \mathcal{E}_{33}+b_{n f} \mathcal{F}_{31}+b_{n s} \mathcal{F}_{32}+b_{n t} \mathcal{F}_{33}\right] P_{n}(\cos \theta),
\end{gathered}
$$

where

$$
\begin{gathered}
\mathcal{E}_{31}=-\left\{-\kappa_{f} r h_{n+1}^{(1)}\left(\kappa_{f} r\right)+n h_{n}^{(1)}\left(\kappa_{f} r\right)\right\} A_{f 2}, \\
\mathcal{E}_{32}=-\left\{-\kappa_{s} r h_{n+1}^{(1)}\left(\kappa_{s} r\right)+n h_{n}^{(1)}\left(\kappa_{s} r\right)\right\} A_{s 2}, \\
\mathcal{E}_{33}=-\frac{\rho_{12}}{\rho_{22}}\left\{n(n+1) h_{n}^{(1)}\left(k_{t} r\right)\right\}, \\
\mathcal{F}_{31}=-\left\{-\kappa_{f} r j_{n+1}\left(\kappa_{f} r\right)+n j_{n}\left(\kappa_{f} r\right)\right\} A_{f 2}, \\
\mathcal{F}_{32}=-\left\{-\kappa_{s} r j_{n+1}\left(\kappa_{s} r\right)+n j_{n}\left(\kappa_{s} r\right)\right\} A_{s 2},
\end{gathered}
$$


Recent Progress in Materials 2021; 3(2), doi:10.21926/rpm.2102020

$$
\mathcal{F}_{33}=-\frac{\rho_{12}}{\rho_{22}} n(n+1) j_{n}\left(k_{t} r\right)
$$

with

$$
A_{f 2}=\frac{A_{f} \kappa_{f}^{2}}{\omega \sqrt{\rho_{22}}}+\frac{\rho_{12}}{\rho_{22}}, A_{s 2}=\frac{A_{s} \kappa_{s}^{2}}{\omega \sqrt{\rho_{22}}}+\frac{\rho_{12}}{\rho_{22}},
$$

Now incident waves are

$$
\begin{gathered}
\phi_{f}^{(i)}=\phi_{0 f}^{(i)} \sum_{n=0}^{\infty}(2 n+1)\left\{1+e^{-2 i \kappa_{f} d}(-1)^{n}\right\} j_{n}\left(\kappa_{f} r\right) P_{n}(\cos \theta), \\
\phi_{s}^{(i)}=\phi_{0 s}^{(i)} \sum_{n=0}^{\infty}(2 n+1)\left\{1+e^{-2 i \kappa_{s} d}(-1)^{n}\right\} j_{n}\left(\kappa_{s} r\right) P_{n}(\cos \theta), \\
u_{r}^{(i)}=\frac{1}{r} \sum_{n=0}^{\infty} \varepsilon_{1} P_{n}(\cos \theta) \\
u_{\theta}^{(i)}=\frac{1}{r} \sum_{n=0}^{\infty} \mathcal{E}_{2} \frac{\partial P_{n}(\cos \theta)}{\partial \theta} \\
v_{r}^{(i)}=\frac{1}{r} \sum_{n=0}^{\infty} \varepsilon_{3} P_{n}(\cos \theta)
\end{gathered}
$$

where

$$
\begin{gathered}
\mathcal{E}_{1}=\varepsilon_{1 f}+\varepsilon_{1 s}, \mathcal{E}_{2}=\varepsilon_{2 f}+\varepsilon_{2 s}, \varepsilon_{3}=\varepsilon_{3 f}+\varepsilon_{3 s}, \\
\mathcal{E}_{1 f}=i^{n}(2 n+1)\left\{1+e^{-2 i \kappa_{f} d}(-1)^{n}\right\}\left\{n j_{n}\left(\kappa_{f} r\right)-\kappa_{f} r j_{n+1}\left(\kappa_{f} r\right)\right\} \phi_{0 f}, \\
\mathcal{E}_{1 s}=i^{n}(2 n+1)\left\{1+e^{-2 i \kappa_{s} d}(-1)^{n}\right\}\left\{n j_{n}\left(\kappa_{s} r\right)-\kappa_{s} r j_{n+1}\left(\kappa_{s} r\right)\right\} \phi_{0 s}, \\
\mathcal{E}_{2 f}=i^{n}(2 n+1)\left\{1+e^{-2 i \kappa_{f} d}(-1)^{n}\right\} j_{n}\left(\kappa_{f} r\right) \phi_{0 f}, \\
\mathcal{E}_{2 s}=i^{n}(2 n+1)\left\{1+e^{-2 i \kappa_{s} d}(-1)^{n}\right\} j_{n}\left(\kappa_{s} r\right) \phi_{0 s}, \\
\mathcal{E}_{3 f}=i^{n}(2 n+1)\left\{1+e^{-2 i \kappa_{f} d}(-1)^{n}\right\}\left\{n j_{n}\left(\kappa_{f} r\right)-\kappa_{f} r j_{n+1}\left(\kappa_{f} r\right)\right\} A_{f 2} \phi_{0 f}, \\
\mathcal{E}_{3 s}=i^{n}(2 n+1)\left\{1+e^{-2 i \kappa_{s} d}(-1)^{n}\right\}\left\{n j_{n}\left(\kappa_{s} r\right)-\kappa_{s} r j_{n+1}\left(\kappa_{s} r\right)\right\} A_{s 2} \phi_{0 s},
\end{gathered}
$$

The boundary conditions at $r=c$ are as follows

$$
\begin{aligned}
& u_{r}^{(i)}+u_{r}^{(r)}=0 \\
& u_{\theta}^{(i)}+u_{\theta}^{(r)}=0
\end{aligned}
$$


Recent Progress in Materials 2021; 3(2), doi:10.21926/rpm.2102020

$$
v_{r}^{(i)}+v_{r}^{(r)}=0,
$$

These will lead to the following equation

$$
E(n) \vec{C}(n)+\mathfrak{F}(n) \vec{B}+\overrightarrow{E_{1}}=0,
$$

Or using relations (21) in the above equation, we find

$$
E \vec{C}(n)+\Im \sum_{m=0}^{\infty} \overrightarrow{C^{\prime}}(m) Q_{m n}+\overrightarrow{E_{1}}=0,
$$

where

$$
\begin{gathered}
\mathrm{E}=\left[\varepsilon_{i j}\right]_{3 \times 3}, \widetilde{F}=\left[\mathcal{F}_{i j}\right]_{3 \times 3} \text { at } r=c \\
\vec{C}(n)=\left[\mathrm{c}_{n f}, \mathrm{c}_{n s}, \mathrm{c}_{n t}\right]^{T}, \overrightarrow{C^{\prime}}(n)=\left[\mathrm{c}_{n f}^{\prime}, \mathrm{c}_{n s}^{\prime}, \mathrm{c}^{\prime}{ }_{n t}\right]^{T}, \\
\vec{B}(n)=\left[\mathrm{b}_{n f}, \mathrm{~b}_{n s}, \mathrm{~b}_{n t}\right]^{T}, \vec{E}_{1}=\left[\varepsilon_{1}, \varepsilon_{2}, \varepsilon_{2}\right]^{T},
\end{gathered}
$$

After using the backward translational theorem to equation (19)

$$
h_{q}^{(1)}(k r) P_{q}(\cos \theta)=\sum_{n=0}^{\infty} Q_{0 q 0 n}^{(2)}(2 d) j_{n}\left(k r^{\prime}\right) P_{n}\left(\cos \theta^{\prime}\right)(-1)^{q+n},
$$

from the second sphere, similarly, we obtain following relation

$$
E(n) \vec{C}^{\prime}(n)+\mathfrak{F}(n) \vec{B}^{\prime}(n)+\overrightarrow{E_{1}}(-1)^{n}=0,
$$

Solving for $\vec{C}^{\prime}(n)$, we find

$$
\vec{C}^{\prime}(\mathrm{n})=-E^{-1} \mathfrak{F}(n) \sum_{m=0}^{\infty} \vec{C}(m) Q_{m n}(-1)^{m+n}-E^{-1}(n) \overrightarrow{E_{1}}(n)(-1)^{n},
$$

Therefore, if we substitute from equation (28) into equation (27), we obtain

$$
E(n) \vec{C}(\mathrm{n})-\mathfrak{F}(n) \sum_{\ell=0}^{\infty} \mathrm{A}(\mathrm{n}, \ell) \vec{C}(\ell)-\mathfrak{F}(n) \mathrm{G}(n)+\overrightarrow{E_{1}}(n)=0,
$$

where

$$
\begin{gathered}
A(\mathrm{n}, \ell)=(-1)^{\ell} \sum_{m=0}^{\infty} E^{-1}(\mathrm{~m}) \mathfrak{F}(m) Q_{\ell m} Q_{m n}(-1)^{m}, \\
G(n)=\sum_{m=0}^{\infty} E^{-1}(m) \overrightarrow{E_{1}}(m) Q_{m n}(-1)^{m}
\end{gathered}
$$


Theorem. The solution $\vec{C}(n)$ to equation (29) exists and is unique.

Equation (29) can be written as

$$
\vec{C}(n)=\sum_{k=0}^{\infty} B(n, k) \vec{C}(k)+\vec{F}(n)
$$

where

$$
\begin{gathered}
B(n, k)=E^{-1}(n) \mathfrak{F}(n) A(n, k) \\
\vec{F}(n)=E^{-1}(n)\left\{\mathfrak{F}(n) G(n)-\overrightarrow{E_{1}}(n)\right\}
\end{gathered}
$$

Let us seek the solution of equation (28) by a method of successive approximations. For the zeroorder approximation, let us take

$$
\vec{C}^{(0)}(n)=\vec{F}(n)
$$

Let us substitute the zero-order approximation in the righthand side of equation (28) and take the resulting expression as the first-order approximation

$$
\vec{C}^{(1)}(n)=\vec{F}(n)+\sum_{k=0}^{\infty} B(n, k) \vec{F}(k),
$$

Moreover

$$
\begin{gathered}
\vec{C}^{(2)}(n)=\vec{F}(n)+\sum_{k=0}^{\infty} B(n, k) \vec{C}^{(1)}(k) \\
=\vec{F}(n)+\sum_{k=0}^{\infty} B(n, k) \vec{F}(k)+\sum_{k=0}^{\infty} \sum_{\ell=0}^{\infty} B(n, k) B(k, \ell) \vec{F}(\ell) \\
=\vec{F}(n)+\sum_{k=0}^{\infty} B(n, k) \vec{F}(k)+\sum_{\ell=0}^{\infty} B_{2}(n, \ell) \vec{F}(\ell),
\end{gathered}
$$

In the above equation, we write, for brevity

$$
B_{2}(n, \ell)=\sum_{k=0}^{\infty} B(n, k) B(k, \ell)
$$

Similarly we find:

$$
\vec{C}^{(3)}(n)=\vec{F}(n)+\sum_{j=0}^{\infty}\left\{B_{1}(n, j)+B_{2}(n, j)+B_{3}(n, j)\right\} \vec{F}(j),
$$

where 


$$
B_{3}(n, j)=\sum_{\ell=0}^{\infty} B(n, \ell) B_{2}(\ell, j)
$$

In general,

$$
\vec{C}^{(m)}(n)=\vec{F}(n)+\sum_{k=1}^{m} \sum_{j=0}^{\infty} B_{k}(n, j) \vec{F}(j),
$$

where $B_{k}(n, j)$ is determined by the recurrence relationship, given by:

$$
B_{1}(n, j)=B(n, j), B_{k}(n, j)=\sum_{\ell=0}^{\infty} B(n, \ell) B_{k-1}(\ell, j) .
$$

Assuming that the approximations do converge and are proceeding to the limit in equation (31), we can obtain a solution of equation (28) and express it in the form of an infinite series

$$
\vec{C}(n)=\vec{F}(n)+\sum_{k=1}^{\infty} \sum_{j=0}^{\infty} B_{k}(n, j) \vec{F}(j) .
$$

Hence, we can show that the series (32) converges and also has a unique limit when the radius of the spheres is small.

Here norms are defined as:

$$
\|\mathrm{E}\|=\max _{i, j}\left\{\varepsilon_{i j}\right\},\|\vec{C}(n)\|=\max \left\{\mathrm{c}_{n f}, \mathrm{c}_{n s}, \mathrm{c}_{n t}\right\},\|\mathfrak{F}(n)\|=\max _{i, j}\left\{\mathcal{F}_{i j}\right\} \text { so on. }
$$

For small values of $r[16,17]$ :

$$
\begin{gathered}
h_{n}^{(1)}(r)=\sqrt{\frac{\pi}{2 r}} H_{n+\frac{1}{2}}^{(1)}(r)=-i \frac{1 \cdot 3 \cdot 5 \cdots(2 n-1)}{r^{n+1}}\left(1-\frac{r^{2}}{2(1-2 n)}+\cdots\right)+\cdots, \\
j_{n}(r)=\sqrt{\frac{\pi}{2 r}} J_{n+\frac{1}{2}}(r)=\frac{r^{n}}{1 \cdot 3 \cdot 5 \cdots(2 n+1)}\left(1-\frac{r^{2}}{2(3+2 n)}+\cdots\right) .
\end{gathered}
$$

By an elaborate work on the above equations, we find:

$$
\begin{gathered}
\|A(n, k)\| \leq \sum_{m=0}^{\infty}\left\|E^{-1}(m)\right\|\|F(m)\|\left\|Q_{k m}\right\|\left\|Q_{m n}\right\| \\
<\sum_{m=0}^{\infty} \frac{r^{m-1} m}{1 \cdot 3 \cdots(2 m-1)} c_{1}^{m+1} c_{2} \frac{r^{m}}{1 \cdot 3 \cdots(2 m+1)} m(m+1) c_{1}^{m} c_{3} K k K m \\
<K_{1} k \sum_{m=0}^{\infty}\left(\frac{r^{m-1}}{1 \cdot 3 \cdots(2 m-1)}\right)^{2} r c_{1}^{2 m+1} c_{2} c_{3} \frac{m^{2}(m+1)}{2 m+1}<K_{2} k
\end{gathered}
$$


Here, we used $\left\|Q_{k m}\right\|<K k$ [12] in equation (36)

Thus,

$$
\begin{aligned}
& \|B(n, k)\|<\left\|E^{-1}(n)\right\|\|F(n)\|\|A(n, k)\|<\frac{r^{n-1} n}{1 \cdot 3 \cdots(2 n-1)} c_{1}^{n+1} c_{2} \\
& \frac{r^{n} n(n+1)}{1 \cdot 3 \cdots(2 n+1)} c_{1}^{n} c_{3} K_{2} k<\frac{r^{2 n-1} c_{1}^{2 n+1} c_{2} c_{3}}{\{1 \cdot 3 \cdots(2 n-1)\}^{2}} \frac{n^{2}(n+1)}{2 n+1} K_{3} k, \\
& \left\|B_{2}(n, j)\right\|=\sum_{\ell=0}^{\infty}\|B(n, \ell)\|\|B(\ell, j)\| \\
& <\frac{r^{2 n-1} c_{1}^{n+1} c_{2} c_{3}}{\{1 \cdot 3 \cdots(2 n-1)\}^{2}} \frac{n^{2}(n+1)}{2 n+1} K_{3} j \sum_{\ell=0}^{\infty} \frac{r^{2 \ell-1} c_{1}^{2 \ell+1} c_{2} c_{3}}{\{1 \cdot 3 \cdots(2 \ell-1)\}^{2}} \frac{\ell^{2}(\ell+1)}{2 \ell+1} K_{3} \ell< \\
& \|B(n, j)\| c_{4},
\end{aligned}
$$

where

$$
c_{4}=\sum_{\ell=0}^{\infty} \frac{r^{2 \ell-1} c_{1}^{2 \ell+1}}{\{1 \cdot 3 \cdots(2 \ell-1)\}^{2}} \frac{\ell^{2}(\ell+1)}{2 \ell+1} K_{3}<\frac{r C}{\left(1-r^{2} c_{1}^{2}\right)^{2}}<1
$$

by choosing a value of $r$ that is small enough to satisfy the above inequality.

$$
\left\|B_{3}(n, j)\right\|<\sum_{\ell=0}^{\infty}\left\|B_{2}(n, \ell) B(\ell, j)\right\| \leq c_{4} \sum_{\ell=0}^{\infty}\|B(n, \ell)\|\|B(\ell, j)\| \leq c_{4}^{2}\|B(n, j)\| .
$$

Generally,

$$
\left\|B_{m}(n, j)\right\| \leq c_{4}^{m-1}\|B(n, j)\| \text {. }
$$

Thus from (32), we have:

$\|\vec{C}(n)\| \leq\|\vec{F}(n)\|+\sum_{k=1}^{\infty} \sum_{j=0}^{\infty}\left\|B_{k}(n, j)\right\|\|\vec{F}(j)\| \leq\|\vec{F}(n)\|+\sum_{k=1}^{\infty} c_{4}^{k-1} \sum_{j=0}^{\infty}\|B(n, j)\|\|\vec{F}(j)\|$.

Equation (37) is convergent, if we choose a small enough value of. From equation (31),

$$
\begin{gathered}
\lim _{m \rightarrow \infty}\left\|\vec{C}^{(m)}(n)-\vec{C}^{(m-1)}(n)\right\|=\lim _{m \rightarrow \infty}\left\|\sum_{j=0}^{\infty} B_{m}(n, j) \vec{F}(j)\right\| \leq \lim _{m \rightarrow \infty} c_{4}^{m-1} \sum_{j=0}^{\infty}\|B(n, j)\|\|\vec{F}(j)\| \\
=0 .
\end{gathered}
$$

Thus, the solution is unique.

\section{Discussions and Conclusion}

In this paper, we have considered the problem of the scattering of plane waves by a rigid inclusion in a poroelastic half space by an image method. This problem can be considered equal to the problem concerning two identical spheres in an infinite medium. Here we have discussed the existence and the uniqueness of the solution. We have also concluded that the results obtained in 
this paper will be useful for the treatment of osteoporosis, which affects elderly people, and is also a threat for potential astronauts.

\section{Author Contributions}

Dr Lee did all the research work of this study.

\section{Competing Interests}

The authors have declared that no competing interests exist.

\section{References}

1. Haire TJ, Langton CM. Biot theory: A review of its application to ultrasound propagation through cancellous bone. Bone. 1999; 24: 291-295.

2. Lakes R, Yoon HS, Katz JL. Ultrasonic wave propagation and attenuation in wet bone. J Biomed Eng. 1986; 8: 143-148.

3. Lakes R, Yoon HS, Katz JL. Slow compressional wave propagation in wet human cortical bone. Science. 1983; 220: 513-515.

4. Lauriks W, Thoen J, Van Ashbroek I, Lowt G, Van der Perre G. Propagation of ultrasonic pulses through trabecular bone. J Phys IV France. 1994; 4: 1255-1258.

5. Wilson JL. Ultrasonic wave propagation in cancellous bone and cortical bone: Prediction of some experimental results by Biot's theory. J Acoust Soc Am. 1992; 91: 1106-1112.

6. Buchanan JL, Gilbert RP. Determination of the parameters of cancellous bone using high frequency acoustic measurements. Math Comput Model. 2007; 45: 281-308.

7. Buchanan JL, Gilbert RP. Determination of the parameters of cancellous bone using high frequency acoustic measurements II: Inverse problems. J Comput Acoust. 2007; 15: 199-220.

8. Gilbert RP, Panchenko A. Acoustics of a stratified poroelastic composite. Z Anal Anwend. 1999; 18: 977-1001.

9. Gilbert RP, Panchenko A, Vasilic A. Homogenizing the acoustics of cancellous bone with an interstitial non-newtonian fluid. Nonlinear Anal Theory Methods Appl. 2011; 74: 1005-1018.

10. Lee DS. Scattering of plane compressional waves in a poroelastic medium having two rigid spherical inclusions. Appl Anal. 2019; 100: 879-892.

11. Hosokawa A, Otani T. Ultrasonic wave propagation in bovine cancellous bone. J Acoust Soc Am. 1997; 101: 558-562.

12. Gaunaurd GC, Huang $H$. Acoustic scattering by a spherical body near a plane boundary. J Acoust Soc Am. 1994; 96: 2526-2536.

13. Biot MA. Theory of Propagation of Elastic Waves in a Fluid-Saturated Porous Solid. I. LowFrequency Range. J Acoust Soc Am. 1956; 28: 168-178.

14. Biot MA. Theory of Propagation of Elastic Waves in a Fluid-Saturated Porous Solid. II. Higher Frequency Range. J Acoust Soc Am. 1956; 28: 179-191.

15. Zimmerman C, Stern M. Scattering of plane compressional waves by spherical inclusions in a poroelastic medium. J Acoust Soc Am. 1993; 94: 527-536.

16. Watson GN. A Treatise on the Theory of Bessel Functions. 2nd ed. Cambridge: Cambridge University Press; 1962. 
17. Abramowitz, M., and Stegun, I., Handbook of mathematical functions: With formulas, graphs, and mathematical tables. New York: Dover Publications; 1972.

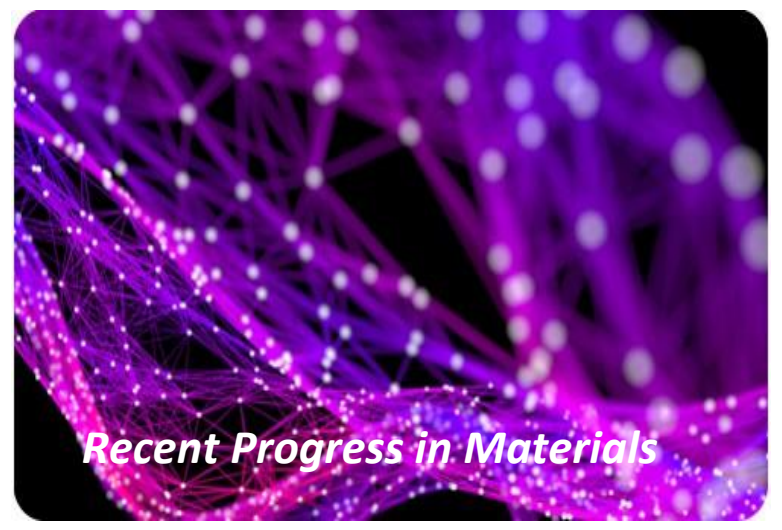

Enjoy Recent Progress in Materials by:

1. Submitting a manuscript

2. Joining in volunteer reviewer bank

3. Joining Editorial Board

4. Guest editing a special issue

For more details, please visit:

http://www.lidsen.com/journals/rpm 